\title{
Panel 2
}

\section{Are Programmable Networks Unmanageable?}

\author{
Organizer: Morris Sloman \\ Imperial College, London, U.K.
}

\begin{abstract}
- active networks

- management by delegation

- mobile agents

- intelligent agents

- policy interpretation
\end{abstract}

There is a need to be able to program network components to adapt to application requirements for quality of service, specialized application dependent routing, to increase efficiency, to support mobility and sophisticated management functionality. There are a number of different approaches to providing programmability:

However there are a number of questions about the manageability of programmable networks.

- If the network is constantly being reprogrammed is it inherently unmanageable?

- Are these technologies so powerful that they are a security risk?

- Who should be permitted to program the network - users or only managers?

- Who are the 'users'?

- How do we charge 'users' for execution of programs within the network?

The panel will discuss these questions and the panelists will each provide a different view-point on the above issues. 